Article

\title{
A Self-Aligned a-IGZO Thin-Film Transistor Using a New Two-Photo-Mask Process with a Continuous Etching Scheme
}

\author{
Ching-Lin Fan ${ }^{1,2, *}$, Ming-Chi Shang ${ }^{1}$, Bo-Jyun Li ${ }^{1}$, Yu-Zuo Lin ${ }^{2}$, Shea-Jue Wang ${ }^{3}$ and \\ Win-Der Lee ${ }^{4}$
}

1 Graduate Institute of Electro-Optical Engineering, National Taiwan University of Science and Technology, Taipei City 106, Taiwan; E-Mails: d10019004@mail.ntust.edu.tw (M.-C.S.); m10019004@mail.ntust.edu.tw (B.-J.L.)

2 Department of Electronic Engineering, National Taiwan University of Science and Technology, Taipei City 106, Taiwan; E-Mail: D9802312@mail.ntust.edu.tw

3 Institute of Materials Science and Engineering, National Taipei University of Technology, Taipei City 106, Taiwan; E-Mail: sjwang@ntut.edu.tw

4 Department of Electrical Engineering, Lee-Ming Institute of Technology, New Taipei City 243, Taiwan; E-Mail: leewd@mail.lit.edu.tw

* Author to whom correspondence should be addressed; E-Mail: clfan@mail.ntust.edu.tw; Tel.: +886-2-2737-6374; Fax: +886-2-2737-6424.

Received: 26 June 2014; in revised form: 24 July 2014 / Accepted: 30 July 2014 /

Published: 11 August 2014

\begin{abstract}
Minimizing the parasitic capacitance and the number of photo-masks can improve operational speed and reduce fabrication costs. Therefore, in this study, a new two-photo-mask process is proposed that exhibits a self-aligned structure without an etching-stop layer. Combining the backside-ultraviolet (BUV) exposure and backside-lift-off (BLO) schemes can not only prevent the damage when etching the source/drain (S/D) electrodes but also reduce the number of photo-masks required during fabrication and minimize the parasitic capacitance with the decreasing of gate overlap length at same time. Compared with traditional fabrication processes, the proposed process yields that thin-film transistors (TFTs) exhibit comparable field-effect mobility $\left(9.5 \mathrm{~cm}^{2} / \mathrm{V} \cdot \mathrm{s}\right)$, threshold voltage $(3.39 \mathrm{~V})$, and subthreshold swing $(0.3 \mathrm{~V} /$ decade). The delay time of an inverter fabricated using the proposed process was considerably decreased.
\end{abstract}


Keywords: amorphous indium-gallium-zinc-oxide (a-IGZO); back-side exposure; self-aligned process; thin-film transistor (TFT); two-photo-mask process; backside-lift-off (BLO)

\section{Introduction}

Numerous recent studies have focused on oxide semiconductors, such as amorphous indium-gallium-zinc oxide (a-IGZO). Because of their high mobility and transparency, these semiconductors have been applied as active channel layers in thin-film transistors (TFTs) [1-3]. Regarding traditional silicon-based TFTs, amorphous silicon (a-Si:H) exhibits low carrier mobility $\left(0.5-1 \mathrm{~cm}^{2} / \mathrm{V} \cdot \mathrm{s}\right)$, whereas polycrystalline silicon (poly-Si) requires high-temperature fabrication processes $\left(>500{ }^{\circ} \mathrm{C}\right)[4,5]$. Conversely, a-IGZO TFTs can be fabricated on plastic substrates at low temperatures and exhibit excellent electrical characteristics [6,7].

The a-IGZO TFTs that are employed in displays are typically fabricated using back-channel-etching structure and five photomasks, including the definition of an etching-stop (ES) layer to protect the a-IGZO active layer from damage caused by etching the source/drain (S/D) electrodes [8]. However, thin-film transistors (TFTs) that involve an ES require a misalignment margin for the ES to ensure the good contact between the S/D and the induced channel; thus, high parasitic capacitances which between the source/drain (S/D) electrodes and gate electrode $\left(C_{\mathrm{gd}}, C_{\mathrm{gs}}\right)$ could occur, decreasing the operational speed of the TFT circuit [9].

To reduce the parasitic capacitance of TFTs with ES, Geng et al. proposed a self-aligned process employing backside-ultraviolet (BUV) exposure through a metal-gate-electrode to define the ES area, thereby reducing the misalignment margin [10]. However, four or five-photo-masks were used during fabrication. To reduce the fabrication costs and prevent hydrogen-based material from affecting the a-IGZO active layer during ES deposition [11], Uhm et al. proposed a two-photo-mask scheme that employed a gray-tone photomask to fabricate TFT devices [12]; however, the lack of an ES layer can cause damage to the a-IGZO active island when etching the $\mathrm{S} / \mathrm{D}$ electrodes. In addition, the ZnO TFT with three photomasks was also proposed [13]. However, the continuous etching of S/D metal, IGZO and gate insulator were not considered in that report. The Table 1 lists the comparisons between these reports and this study.

Table 1. Summary of indium-gallium-zinc oxide (IGZO)-based thin-film transistors (TFTs) using different process.

\begin{tabular}{cccccc}
\hline Reference & $\begin{array}{c}\text { Channel } \\
\text { material }\end{array}$ & Self-aligned & $\begin{array}{c}\text { Mask } \\
\text { number }\end{array}$ & $\begin{array}{c}\text { S/D Etching } \\
\text { damage }\end{array}$ & $\begin{array}{c}\text { a-IGZO Degradation due to } \\
\text { ES-layer deposition }\end{array}$ \\
\hline$[10]$ & GGZO & Yes & 4 & No & Yes \\
{$[12]$} & IGZO & No & 2 & Yes & No \\
{$[13]$} & ZnO & Yes & 3 & No & No \\
this work & IGZO & Yes & 2 & No & No \\
\hline
\end{tabular}




\section{Device Fabrication}

Figure 1 shows the proposed two-mask process for fabricating a-IGZO TFTs. A 160-nm-thick Ti layer was first deposited onto a glass substrate by using thermal evaporation, and then patterned to form the gate electrode by the first photomask. Subsequently, a 200 -nm-thick silicon dioxide $\left(\mathrm{SiO}_{2}\right)$ was deposited using plasma enhanced chemical vapor deposition at $300{ }^{\circ} \mathrm{C}$, forming the gate insulator. Subsequently, a 20-nm a-IGZO layer was deposited at $200{ }^{\circ} \mathrm{C}$ by a radio frequency (RF) sputtering system using a target of $\mathrm{In}: \mathrm{Ga}: \mathrm{Zn}=1: 1: 1$ in atomic ratio. The backside-lift-off (BLO) process is detailed as follows. First, a photo-resist (PR) was spin-coated onto IGZO and subjected to BUV exposure through the Ti gate as a photomask, as shown in Figure 1. Second, a 350-nm-thick Indium-Tin Oxide (ITO) was deposited using RF sputtering. Subsequently, the BLO scheme was applied to define the channel length of the self-aligned structure. Following the BLO process, the second photomask were used to define the channel width. Reactive-ion etching (RIE) with $\mathrm{CF}_{4}$ gas was used to continuously etch ITO, IGZO and $\mathrm{SiO}_{2}$ under the pressure of 80 mtorr. Finally, the devices were annealed at $200{ }^{\circ} \mathrm{C}$ for $30 \mathrm{~min}$ in a vacuum chamber. To compare the proposed devices, we used a traditional four-photo-mask process to fabricate devices that exhibited various overlap lengths between the gates and S/D electrodes [11].

Figure 1. Two-Mask process flow of amorphous indium-gallium-zinc-oxide (a-IGZO) thin-film transistors (TFTs) with self-aligned structure.

\section{Step 1: Gate patterning ( $\left(^{\text {st }}\right.$ Mask $)$}

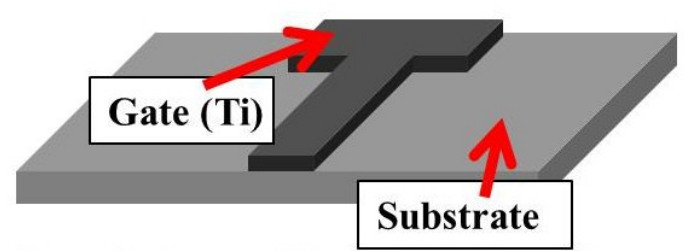

Step 2: Deposition of $\mathrm{SiO}_{2}$ and

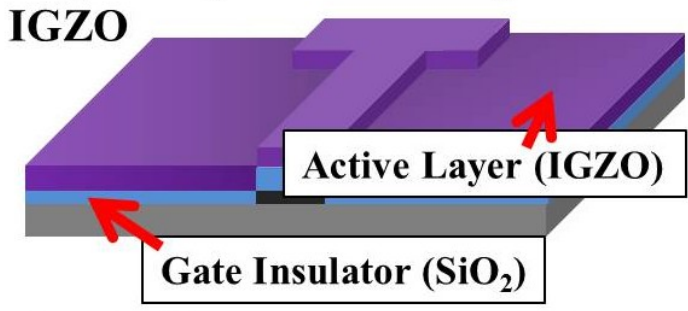

Step 3: Photo-resistance coating

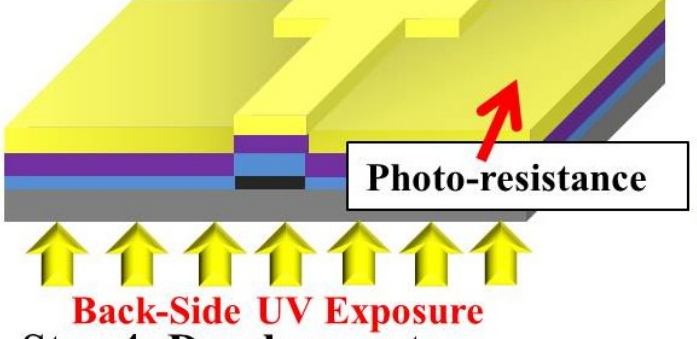

Step 4: Development

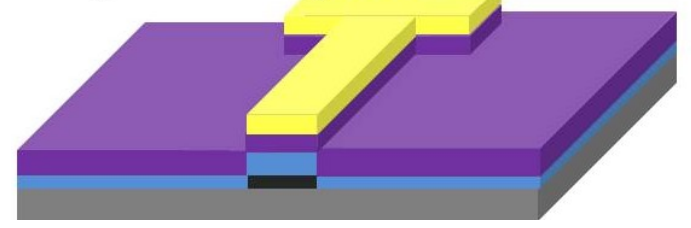

Step 5: Deposition of ITO

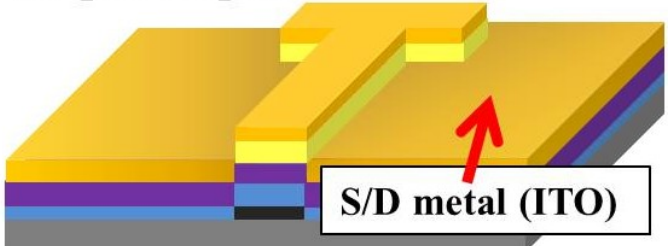

Step 6: Lift-off process
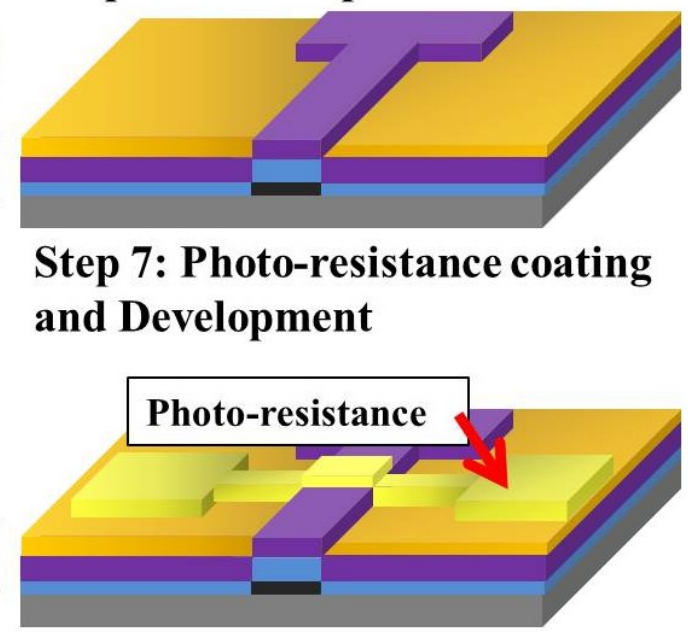

Step 8: Defining the channel width and contact hole ( $2^{\text {nd }}$ Mask)

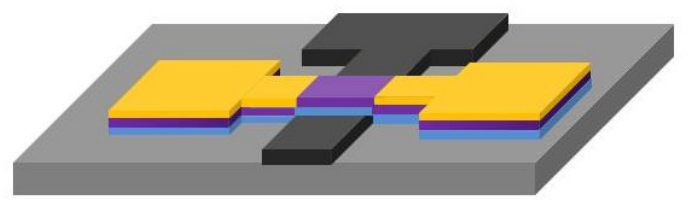




\section{Results and Discussion}

In this study, a new two-photo-mask process with continuous-etching scheme was proposed for fabricating a-IGZO TFTs that exhibit self-aligned structures without ES layers. The ITO metal was designed as $\mathrm{S} / \mathrm{D}$ metal to meet the requirement of the continuous etching process. Thus, S/D metal, IGZO and gate insulator can simultaneously be etched. Combining the BUV exposure and backside-lift-off (BLO) schemes can not only prevent the damage when etching the S/D electrodes but also reduce the number of photo-masks required during fabrication and minimize the parasitic capacitance at same time.

Figure 2a,b shows the transfer and output characteristics of the proposed a-IGZO TFTs with self-aligned structure for channel width (W) of $50 \mu \mathrm{m}$ and channel length (L) of $50 \mu \mathrm{m}$, respectively. A total of 20 devices were measured at various positions across the substrate by using a semiconductor parameter analyzer (HP4145B Hewlett Packard, Palo Alto, CA, USA). The extracted saturation field-effect mobility $\left(\mu_{\mathrm{sat}}\right)$, threshold voltage $\left(V_{\mathrm{T}}\right)$, subthreshold swing $(S)$, and on-off current ratio $\left(I_{\text {on }} / I_{\text {off }}\right)$ are $9.50 \mathrm{~cm}^{2} / \mathrm{V} \cdot \mathrm{s}, 3.39 \mathrm{~V}, 0.3 \mathrm{~V} /$ decade, and $4 \times 10^{7}$, respectively. $\mu_{\text {sat }}$ and $V_{\mathrm{T}}$ are extracted from the slope and linear extrapolations of the plot of the square root of the drain current as a function of the gate voltage, respectively [14-16]. Moreover, the output characteristics show that current crowding did not occur in the linear region to reveal the contact resistance of the S/D regions can satisfy the requirement of transportation current [17].

Figure 2. (a) Transfer characteristic $\left(V_{\mathrm{DS}}=15 \mathrm{~V}\right)$ and (b) output characteristic of a-IGZO TFT with self-aligned structure $(W / L=50 \mu \mathrm{m} / 50 \mu \mathrm{m})$.

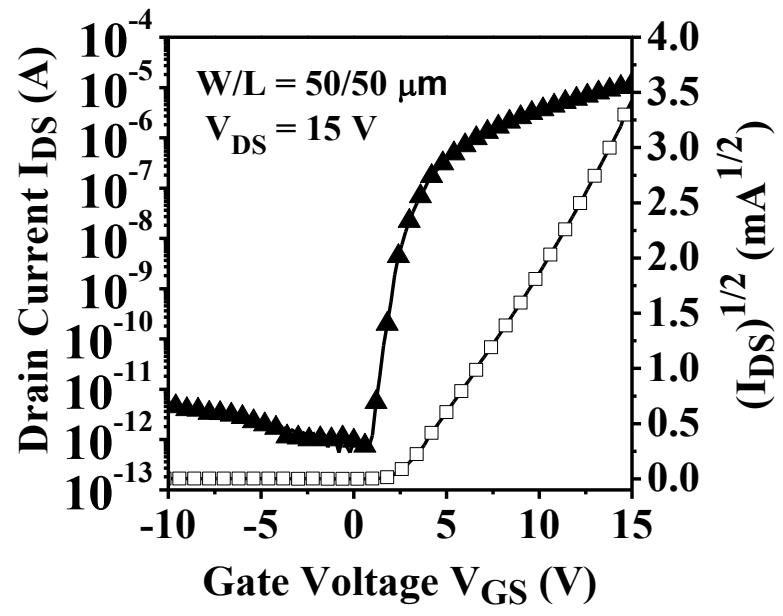

(a)

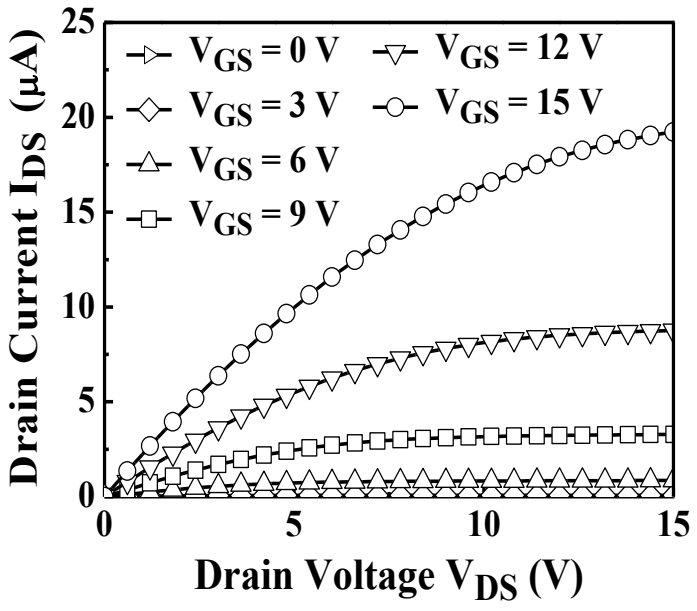

(b)

The devices were fabricated using the traditional four-photo-mask process, yielding various overlap lengths $\left(L_{\mathrm{ov}}=100\right.$ and $\left.200 \mu \mathrm{m}\right)$ between the gate and S/D electrodes. Figure 3 shows optical microscopy images and scanning electron microscopy images of the a-IGZO TFTs, which exhibit self-aligned and overlapping structures. In Figure 3a,b, the overlapping region less than $0.5 \mu \mathrm{m}$ could be observed in scanning electron microscopy images. The overlapping region was clearly observable among the devices fabricated using the traditional four-photo-mask process in Figure 3c, which yielded parasitic capacitance between $\mathrm{S} / \mathrm{D}$ and gate electrodes $\left(C_{\mathrm{gd}}, C_{\mathrm{gs}}\right)$. The parasitic capacitance was proportional to 
the area of overlapping region $\left(W \times L_{\mathrm{ov}}\right)$, yielding increased feed-through voltage, noise, and circuit delay in devices typically used in displays applications [12].

To analyze the parasitic capacitance between the S/D and gate electrodes, the capacitance-voltage of the fabricated a-IGZO TFTs was measured using an Inductance-Resistance-Capacitance (LCR) meter (HP4284A, Hewlett Packard, Palo Alto, CA, USA; Figure 4). The capacitance markedly increased in conjunction with the overlap length $\left(L_{\mathrm{ov}}\right)$ (i.e., the area of overlapping region). The device that was fabricated using the traditional four-photo-mask process (i.e., $L_{\mathrm{ov}}$ of $200 \mu \mathrm{m}$ ) exhibited a minimal capacitance of $11.46 \mathrm{pF}$, which was considerably higher compared with that of the device fabricated using the proposed method $(0.25 \mathrm{pF})$. Hence, the self-aligned structure reduced the parasitical capacitance between the S/D and gate electrodes. To examine the effects of parasitic capacitance, two types of the inverters were fabricated (Figure 5a); the first comprised the proposed two-mask process with self-aligned structures, and the second comprised overlapping structures (both types employed an $\mathrm{N}$-type metal-oxide-semiconductor configuration as the active load). Figure 5a also shows the dynamic state measurements for the inverters. Various square wave frequencies $\left(V_{\mathrm{IN}}\right)$ were input into the inverters, and the output signals ( $V_{\text {OUT }}$ ) were measured using oscilloscope. The rise time of the output signal was defined as the time required by the signal to rise from $10 \%$ to $90 \%$ of the step height; this time is related to the parasitic capacitance of TFTs due to the Resistance-Capacitance (RC) delay for inverter circuit operation. Figure $5 \mathrm{~b}$ indicates that the rise time (delay time) increased in conjunction with the overlap length $\left(L_{\mathrm{ov}}\right)$, (i.e., the parasitic capacitances). Compared with inverter with $L_{\mathrm{ov}}$ of $200 \mu \mathrm{m}$, the inverter that comprised the proposed two-mask schemes with self-aligned structure exhibited a substantial decrease in delay time from 230 to $78 \mu$ s (the input frequency is $500 \mathrm{~Hz}$ ).

Figure 3. (a) Optical microscopy images of a-IGZO TFTs with self-aligned structures;

(b) Scanning electron microscopy images of a-IGZO TFTs with self-aligned structures;

(c) Optical microscopy images of a-IGZO TFTs with overlap structures.

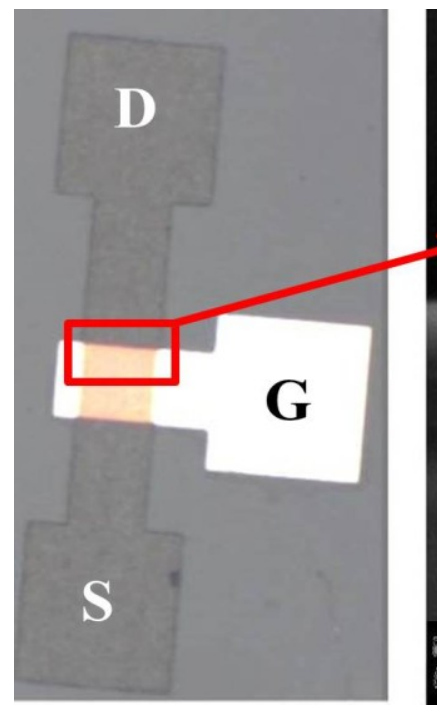

(a)

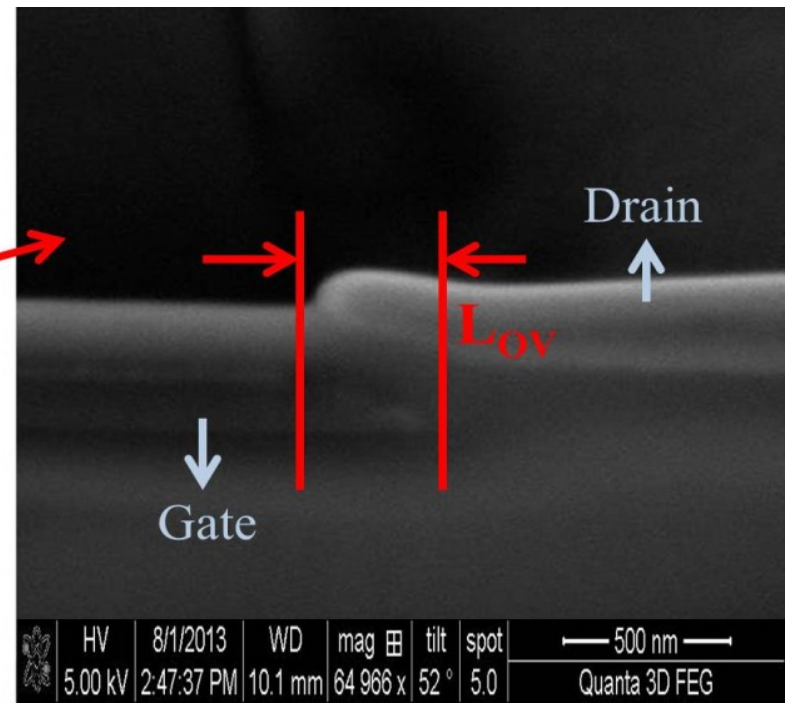

(b)

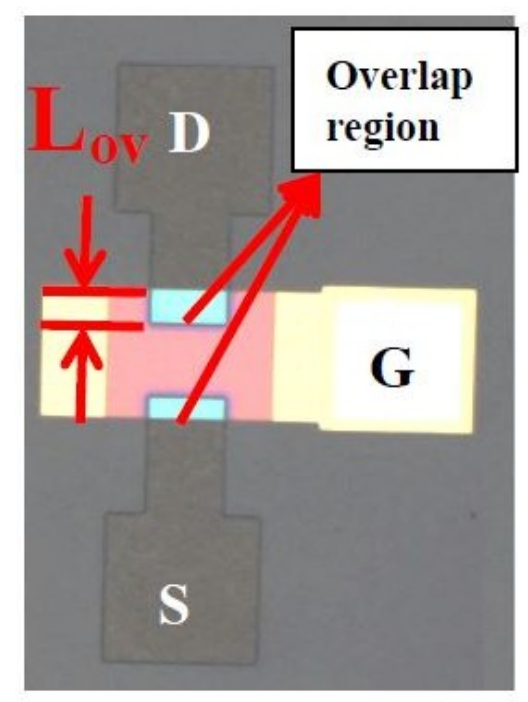

(c) 
Figure 4. Gate-to-source capacitance $\left(C_{\mathrm{gs}}\right)$ of a-IGZO TFTs with self-aligned structure and overlap structure for different $L_{\mathrm{ov}}(W=200 \mu \mathrm{m})$.

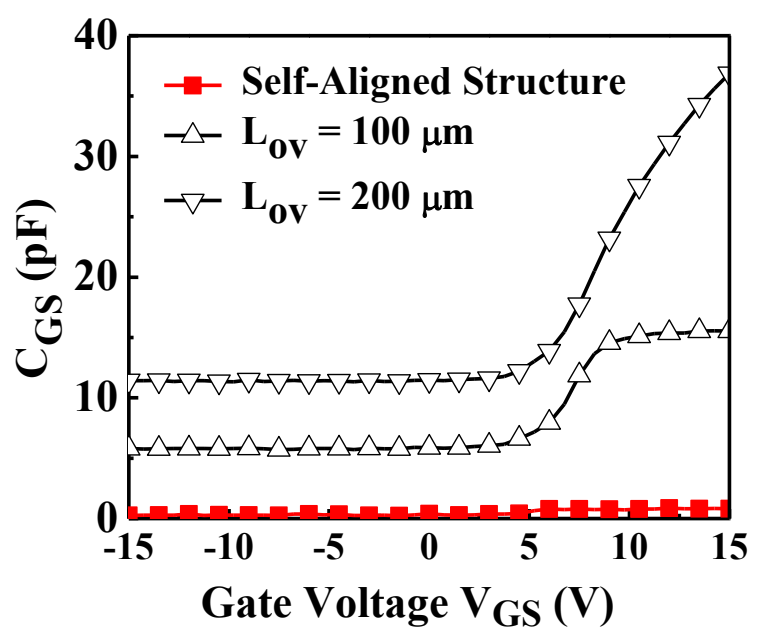

Figure 5. (a) Inverter composed of the fabricated a-IGZO TFTs $\left(V_{\mathrm{DD}}=20 \mathrm{~V}, W_{\text {Drive }}=200 \mu \mathrm{m}\right.$, $L_{\text {Drive }}=10 \mu \mathrm{m}, W_{\text {Load }}=40 \mu \mathrm{m}$, and $L_{\text {Load }}=10 \mu \mathrm{m}$ ); (b) The extracted rise time for inverters composed of a-IGZO TFTs with self-aligned structure and overlap structure for $L_{\mathrm{ov}}=100$ and $200 \mu \mathrm{m}$.

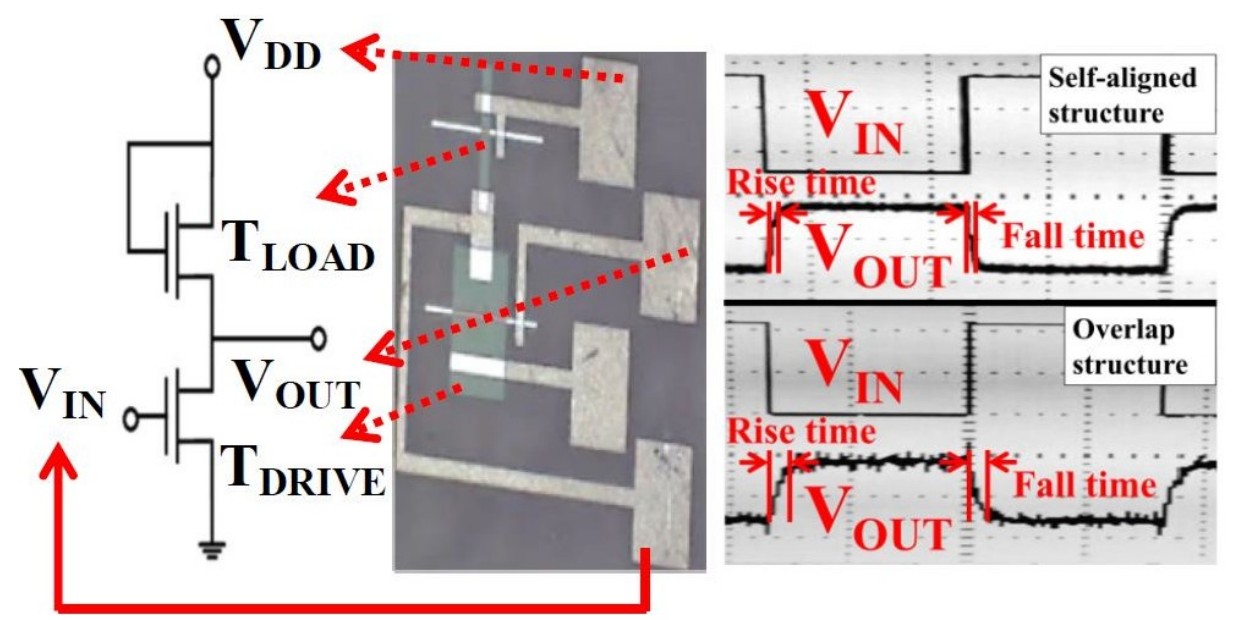

(a)

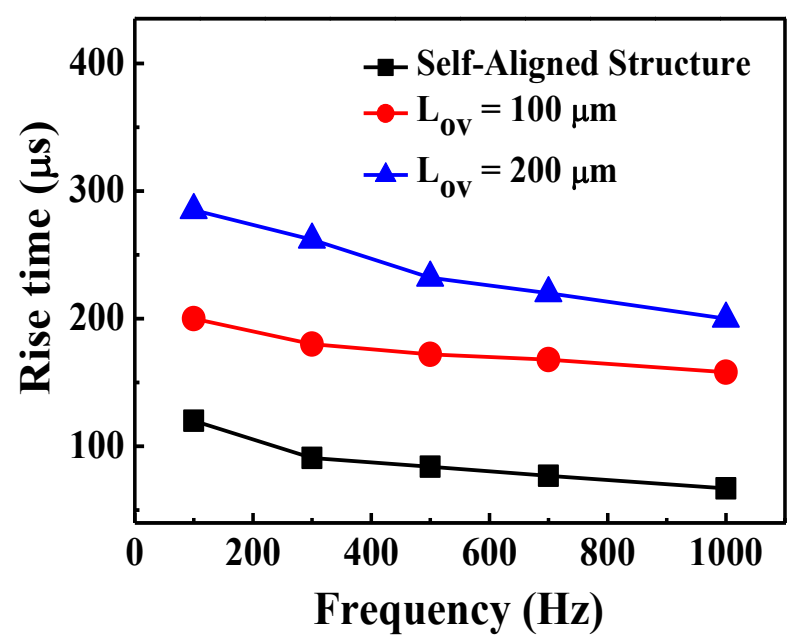

(b) 


\section{Conclusions}

In this study, a novel two-photo-mask process was proposed for fabricating a-IGZO TFTs that exhibit a self-aligned structure. Combining the BUV exposure and BLO schemes reduced both the parasitic capacitance and number of photo-masks required for fabrication. In addition, the proposed devices that lack ES layers yield an undamaged a-IGZO active layer, facilitating superior performance levels (such as a field-effect mobility of $9.5 \mathrm{~cm}^{2} / \mathrm{V} \cdot \mathrm{s}$, a threshold voltage of $3.39 \mathrm{~V}$, and a subthreshold swing of $0.3 \mathrm{~V} /$ decade). Compared with using a traditional four-photo-mask process and no ES layer, the proposed process can be used to fabricate a-IGZO TFTs that exhibit fair performance levels. Moreover, the reduced parasitic capacitance yielded a marked decrease in delay time in an inverter fabricated using the proposed method. Thus, the proposed process is a suitable candidate for use in a-IGZO TFT applications, such as active-matrix organic light-emitting diodes (AMOLED), because it achieves high operational speeds and reduces fabrication costs.

\section{Acknowledgments}

The authors would like to acknowledge the financial support of the National Science Council of Taiwan under contract No. NSC 101-2221-E-011-070 and NSC 102-2221-E-011-112-MY2.

\section{Author Contributions}

Ching-Lin Fan advised the study and work; Ching-Lin Fan and Bo-Jyun Li designed the research; Bo-Jyun Li and Ming-Chi Shang performed the experimental work; Ming-Chi Shang wrote the manuscript. All authors discussed, edited and approved the final version.

\section{Conflicts of Interest}

The authors declare no conflict of interest.

\section{References}

1. Hirao, T.; Furuta, M.; Hiramatsu, T.; Matsuda, T.; Li, C.; Furuta, H.; Hokari, H.; Yoshida, M.; Ishii, H.; Kakegawa, M. Bottom-gate zinc oxide thinfilm transistors (ZnO TFTs) for AM-LCDs. IEEE Trans. Electron Devices 2008, 55, 3136-3142.

2. Paine, D.; Yaglioglu, B.; Beiley, Z.; Lee, S.H. Amorphous IZO based transparent thin film transistors. Thin Solid Films 2008, 516, 5894-5898.

3. Kumomi, H.; Nomura, K.; Kamiya, T.; Hosono, H. Amorphous oxide channel TFTs. Thin Solid Films 2008, 516, 1516-1522.

4. Ukai, Y. TFT-LCD manufacturing technology - Current status and futureprospect. In Proceedings of International Workshop on Physics of Semiconductor Devices, Mumbai, India, 16-20 December 2007; pp. 29-34.

5. Choi, Y.W.; Lee, J.N.; Jang, T.W. Thin-film transistors fabricated with Poly-Si films crystallized at low temperature by microwave annealing. IEEE Electron Device Lett. 1999, 20, 2-4. 
6. Nomura, K.; Ohta, H.; Ueda, K.; Kamiya, T.; Hirano, M.; Hosono, H. Thin-film transistor fabricated in single-crystalline transparent oxide emiconductor. Science 2003, 300, 1269-1272.

7. Nomura, K.; Ohta, H.; Takagi, A.; Kamiya, T.; Hirano, M.; Hosono, H. Room-temperature fabrication of transparent flexible thin-film transistors using amorphous oxide semiconductor. Nature 2004, 432, 488-492.

8. Liu, S.; Yu, M.; Lin, C.; Ho, G.; Cheng, C.; Lai, C.; Lin, C.; King, Y.; Yeh, Y. Influence of passivation layers on characteristics of a-InGaZnO thin-film transistors. IEEE Electron Device Lett. 2011, 32, 161-163.

9. Kodkani, R.M.; Larson, L.E. A $25 \mathrm{GHz}$ quadrature voltage controlled ring oscillator in $0.12 \mu \mathrm{m} \mathrm{Si}$ Ge HBT. In Proceedings of the IEEE Topical Meeting on Silicon Monolithic Integrated Circuits in RF Systems, San Diego, CA, USA, 18-20 January 2006; pp. 383-386.

10. Geng, D.; Kang, D.H.; Jang, J. High-performance amorphous indium-gallium-zinc-oxide thin-film transistor with a self-aligned etch stopper patterned by back-side UV exposure. IEEE Electron Device Lett. 2011, 32, 758-760.

11. Park, J.; Kim, S.; Kim, C. High-performance amorphous gallium indium zinc oxide thin-film transistors through $\mathrm{N}_{2} \mathrm{O}$ plasma passivation. Appl. Phys. Lett. 2008, 93, 053505:1-053505:3.

12. Uhm, H.; Lee, S.; Kim, W.; Park, J. A two-mask process for fabrication of bottom-gate IGZO-based TFTs. IEEE Electron Device Lett. 2012, 33, 543-545.

13. Mourey, D.A.; Zhao, D.A.; Jackson, T.N. Self-aligned-gate ZnO TFT circuits. IEEE Electron Device Lett. 2010, 31, 326-328.

14. Chen, T.L.; Hsu, T.Y.; Lin, H.Y.; Chou, C.H.; Lin, H.H.; Liu, C.W. Enhanced current drive of double-gate $\alpha$-IGZO thin-film transistors. IEEE Electron Device Lett. 2013, 34, 417-419.

15. Kamiya, T.; Nomura, K.; Hosono, H. Present status of amorphous In-Ga-Zn-O thin-film transistors. Sci. Technol. Adv. Mater. 2010, 11, 044305:1-044305:23.

16. Seok, M.J.; Choi, M.H.; Mativenga, M.; Kim, D.Y.; Jang, J. A full-swing a-IGZO TFT-based inverter with a Top-Gate-Bias-Induced depletion load. IEEE Electron Device Lett. 2011, 32, 1089-1091.

17. Han, D.-S.; Kang, Y.-J.; Park, J.-H.; Jeon, H.-T.; Park, J.-W. Influence of molybdenum source/drain electrode contact resistance in amorphous zinc-tin-oxide (a-ZTO) thin film transistors. Mater. Res. Bull. 2014, 2014, doi:10.1016/j.materresbull.2014.05.009.

(C) 2014 by the authors; licensee MDPI, Basel, Switzerland. This article is an open access article distributed under the terms and conditions of the Creative Commons Attribution license (http://creativecommons.org/licenses/by/3.0/). 